\title{
Enhanced buoyancy of active particles in convective flows
}

\author{
Yunyun Li®, ${ }^{1}$ Pulak K. Ghosh, ${ }^{2}$ and Fabio Marchesoni ${ }^{1,3}$ \\ ${ }^{1}$ Center for Phononics and Thermal Energy Science, Shanghai Key Laboratory of Special Artificial Microstructure \\ Materials and Technology, School of Physics Science and Engineering, Tongji University, Shanghai 200092, China \\ ${ }^{2}$ Department of Chemistry, Presidency University, Kolkata 700073, India \\ ${ }^{3}$ Department of Physics, University of Camerino, I-62032 Camerino, Italy
}

(Received 13 July 2021; accepted 3 September 2021; published 14 September 2021)

\begin{abstract}
We numerically investigated the diffusion of a heavy active Brownian particle in a linear periodic array of steady planar counter-rotating convection rolls at high Péclet numbers. We show that, under certain conditions, the particle rises to the surface even if it is denser than the suspension fluid, and floats there for exceedingly long times. Such an apparently counterintuitive phenomenon of "enhanced buoyancy" is a combined effect of gravity, advection, and shear torque.
\end{abstract}

DOI: 10.1103/PhysRevResearch.3.L032065

Introduction. All sorts of counterintuitive phenomena are known to occur in mechanical systems maintained out of equilibrium [1]. Among the best known examples are the inverted (Stephenson-Kapitza and Chelomei) pendulums, namely rigid rods designed to oscillate upside down by vertically shaking their suspension points [2]. In rheological systems, vibrational forces may also lead to intriguing effects, like the sinking of gas bubbles in shaken liquids [3]. Levitation of a fluid layer above air is another surprising example [4], whereby relatively heavy objects can float upside down on the layer's lower interface, as if gravity were inverted [3].

Also apparently defying gravity is the "Brazilian nut effect" [5]. When a box containing one large particle and a mixture of smaller ones is vertically shaken, the large particle can rise to the top, even if it is denser than the others. Segregation of granular materials by size is due to the interplay of external (gravitational and vibrational) and internal (particleparticle and particle-wall) forces and depends on the particle shapes [6].

Correspondingly, when not restricted to a container, a mass moving under nonequilibrium conditions can drift against an external bias, with negative mobility (either differential or absolute). Differential negative mobility is a relatively common phenomenon [7]; it is due to the obstructing action of the suspension medium in response to the driven tracer itself, as summarized by the catchall "getting more by pushing less" [8]. Absolute negative mobility, instead, results from tailored combinations of time memory, spatial asymmetry, and driving fields $[9,10]$. So far, negative mobility has been demonstrated numerically only for particles suspended in periodically mod-

Published by the American Physical Society under the terms of the Creative Commons Attribution 4.0 International license. Further distribution of this work must maintain attribution to the author(s) and the published article's title, journal citation, and DOI. ulated environments and in the presence of either inertia $[9,11]$ or asymmetric confining geometries [10].

In this Letter we investigate a less contrived realization of negative response to an external bias, namely the floating properties of a pointlike heavy active Brownian particle periodically advected in a linear array of convection rolls [12]. In view of a practical realization, we will refer for convenience to the archetypal case of a Janus particle (JP). JPs are strongly idealized artificial microswimmers capable of self-propulsion in an active medium [13,14]. Spherical colloidal particles with two differently coated hemispheres, or "faces," they are designed to harvest environmental energy and convert it into kinetic energy through a variety of microscopic physical-chemical processes $[15,16]$. Our numerical simulations reveal that, when suspended in a steady convection flow, active JPs can rise to the surface and float there, even if they are denser than the suspension fluid. This surprising effect, termed here "enhanced buoyancy" (EB), occurs when self-propulsion and advection speeds grow comparable. The underlying dynamical mechanism results from the interplay of the drag exerted by gravity and advection, and the torque applied by the convective flow shear. However, in contrast with the earlier literature, neither inertia [11] nor taxis [17] are required.

Model. Let us consider an overdamped, pointlike active JP diffusing in the convection array of stream function $\psi(x, y)$ [18-20],

$$
\psi(x, y)=\left(U_{0} L / 2 \pi\right) \sin (2 \pi x / L) \sin (2 \pi y / L),
$$

with lower (upper) edge $y=0(y=L / 2)$, under the drag of its apparent weight (i.e., weight minus buoyant force), $g$ [21]. Its planar dynamics is described by the Langevin equations (LE) $[22,23]$,

$$
\begin{aligned}
\dot{\mathbf{r}} & =\mathbf{u}+\mathbf{v}_{0}(\theta)+\mathbf{F}+\sqrt{D_{0}} \boldsymbol{\xi}(t) \\
\dot{\theta} & =(\alpha / 2) \nabla \times \mathbf{u}+\sqrt{D_{\theta}} \xi_{\theta}(t),
\end{aligned}
$$


where $\mathbf{F}=(0,-g)$ and $\mathbf{u}(x, y)=\left(u_{x}, u_{y}\right)=\left(\partial_{y},-\partial_{x}\right) \psi(x, y)$ is the incompressible advection velocity vector with $\nabla$. $\mathbf{u}=0$. The array unit cell consists of two counter-rotating convection rolls; reflecting boundaries are assumed at the array edges. The random sources, $\boldsymbol{\xi}(t)=\left(\xi_{x}(t), \xi_{y}(t)\right)$ and $\xi_{\theta}(t)$ are stationary, independent, delta-correlated Gaussian noises, $\left\langle\xi_{i}(t) \xi_{j}(0)\right\rangle=2 \delta_{i j} \delta(t)$, with $i=x, y, \theta . \boldsymbol{\xi}(t)$ models equilibrium thermal fluctuations in a homogeneous, isotropic medium. In the notation of Eq. (2), $D_{0}$ coincides with the free passive-particle diffusion constant in the absence of advection. $\xi_{\theta}(t)$ represents the rotational noise affecting the orientation, $\theta$, of the self-propulsion velocity, $\mathbf{v}_{0}(\theta)=$ $v_{0}(\cos \theta, \sin \theta)$. The reciprocal of $D_{\theta}$ coincides with the angular persistence (or correlation) time, $\tau_{\theta}$, of $\mathbf{v}_{0}(\theta)$; accordingly, $l_{\theta}=v_{0} / D_{\theta}$ quantifies the persistence length of the particle's self-propelled random motion. The flow shear exerts a torque on the particle, $\Omega_{\alpha}(x, y)=(\alpha / 2) \nabla \times \mathbf{u}$, proportional to the local fluid vorticity. For simplicity, we adopt Faxén's second law, which, for an ideal no-stick spherical particle, yields $\alpha=$ 1 [24]. Contrary to earlier work [25-27], in the Lagrangian formalism of LE (2), "trajectory overshooting" (i.e, particle trajectories crossing flow streamlines) is caused by the drag terms, $\mathbf{v}_{0}(\theta)$ and $\mathbf{F}$, and not by inertia.

In the high Péclet number regime addressed here, $\mathrm{Pe} \equiv$ $D_{L} / D_{0} \gg 1$, particle diffusion is strongly influenced by advection. The flow parameters define convenient length and time units, respectively, $L$ and $\Omega_{L}^{-1}$, with $\Omega_{L}=2 \pi U_{0} / L$; hence, the advection diffusion constant $D_{L}=U_{0} L / 2 \pi$. Therefore, the only tunable parameters left in our model are the noise strengths, $D_{0}$ (in units of $D_{L}$ ), $D_{\theta}$ (in units of $\Omega_{L}$ ), and the drives $g$ and $v_{0}$ (in units of $U_{0}$ ). The stochastic differential Eqs. (2) were numerically integrated for a single particle by means of a standard Milshtein scheme [28]. Therefore, the results reported here apply to dilute mixtures of active JPs, only, clustering and jamming effects being not accounted for [15]. To ensure numerical stability, the numerical integrations have been performed using a very short time step, $10^{-5}-10^{-4}$.

Results. Numerical evidence of EB is presented in Fig. 1, where the stationary probability density function (pdf), $p(x, y)$, of the advected JP is plotted for increasing values of the self-propulsion speed. The apparent weight, $g$, pushes the particle against the lower array edge, $y=0$. Accordingly, for $v_{0}<U_{0}$, Figs. 1(a) and 1(b), the pdf's tend to accumulate at the bottom of the convection array. Circulation due to advection in the clockwise (right) and counterclockwise (left) rolls, is clearly visible. With increasing $v_{0}$, the particle circulation is restricted to the center-bottom of the rolls. This mechanism is further illustrated by the trajectory samples of Fig. 1(e). A totally different scenario emerges for $v_{0} \gtrsim U_{0}$, Figs. 1(c) and 1(f). The particle either crosses the convection rolls along the lower array edge or gets trapped in symmetric pockets at their center-tops. Most remarkably, the particle sojourns in the vicinity of either edge for much longer time intervals than any model timescale.

Finally, for $v_{0} \gg U_{0}$ the JP switches array edge mostly because of self-propulsion, that is, with time constant $\tau_{\theta}$; gravity and advection grow relatively negligible and $p(x, y)$ tends to accumulate equally along the array edges.

More insight can be gained by inspecting the longitudinal pdf's of Fig. 2, $p(x)$, obtained by integrating $p(x, y)$ over the
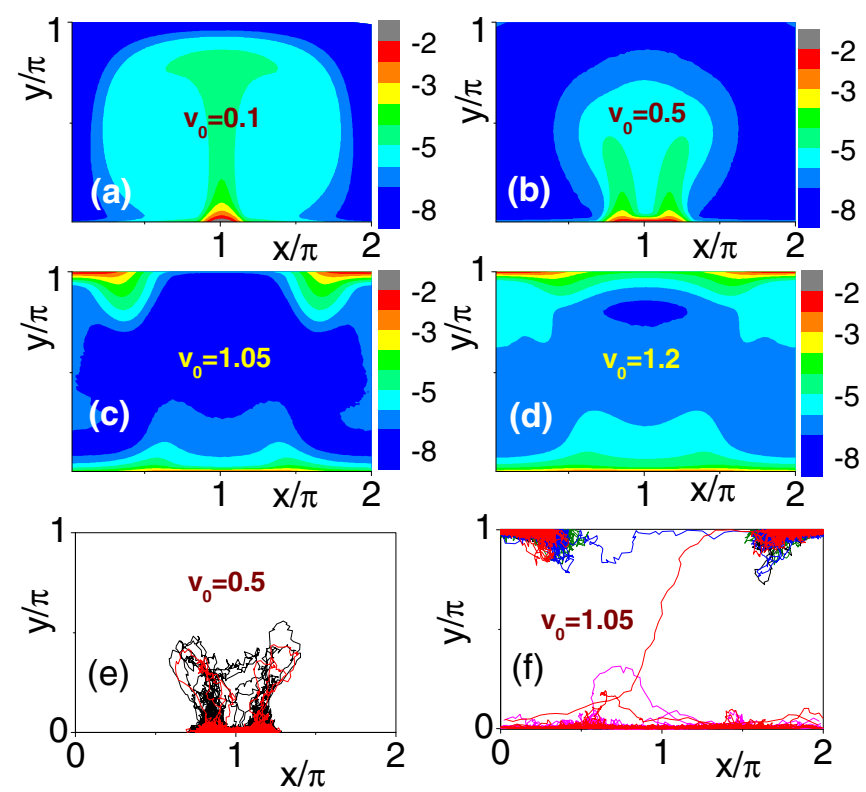

FIG. 1. Spatial distributions, $p(x, y)$, of a JP in the laminar flow of Eq. (1) for $g=0.4$ and different $v_{0}$ [(a)-(d)]. The chart levels are color coded on natural logarithmic scales as indicated. Trajectory samples (2400 time-unit long, drawn in different colors) immediately before (e) and after (f) the EB onset, are reported for a comparison. Other simulation parameters are $D_{0}=D_{\theta}=0.01, U_{0}=1$, and $L=2 \pi$

vertical coordinate $y$. A passive JP, $v_{0}=0$, is advected along the streamlines of the incompressible flow. For $g=0, p(x, y)$ and, therefore, $p(x)$, are uniform. The external bias, $g$, breaks the roll symmetry, until, for $g>g_{c}=4 \sqrt{D_{0} / D_{L}}$, the particle circulation is suppressed [21]. The particle then diffuses along the lower array edge, subjected to the effective washboard potential $V_{0}(x)=U_{0}\langle\cos (2 \pi y / L)\rangle \cos (2 \pi x / L)$, with $\langle\cos (2 \pi y / L)\rangle \sim 1$ for $g>g_{c}$ [21]. As a result, the particle tends to settle at the bottom of the ascending flows $[x=\pi$ in Fig. 2(a)] and $p(x)$ is a periodic function with period $L$. Let us consider now an active JP in the absence of gravity, $g=0$, Fig. 2(b). The system is up-down symmetric and $p(x)$ periodic with period $L / 2$. Indeed, on increasing $v_{0}$, the particle stops circulating inside the rolls [23] and sojourns preferably, with equal probability, at the bottom (top) of the ascending (descending) flows; hence the $p(x)$ peaks at $x=0 \bmod (L / 2)$. As anticipated above, for $v_{0} \gg U_{0}$, the $p(x)$ peak structure vanishes. In the presence of gravity, the interplay of external, $g$, and intrinsic drag, $v_{0}$, impacts the $p(x)$ profile in an unexpected manner, as illustrated in Fig. 2(c): Increasing $v_{0}$ weakens the ascending flow peaks of $p(x)$ [at $x=\pi$ in Fig. 2(a)]; side peaks emerge around $x=0$ and $2 \pi$, which, for $v_{0} \gtrsim U_{0}$, eventually overshoot the central peak. Under these conditions, the advected JP rises to the upper array edge and sits preferably at the top of the descending flows [29].

The occurrence of an EB effect is confirmed by the simulation data of Fig. 3. In Figs. 3(a) and 3(b) we plotted respectively the ratios $p_{x}(0) / p_{x}(\pi)$ and $p_{y}(\pi) / p_{y}(0)$ as functions of the self-propulsion speed for $g>0$. Here $p(y)$ was obtained by integrating $p(x, y)$ over $x$. The ratios' denominators are a measure of the particle accumulation respectively 


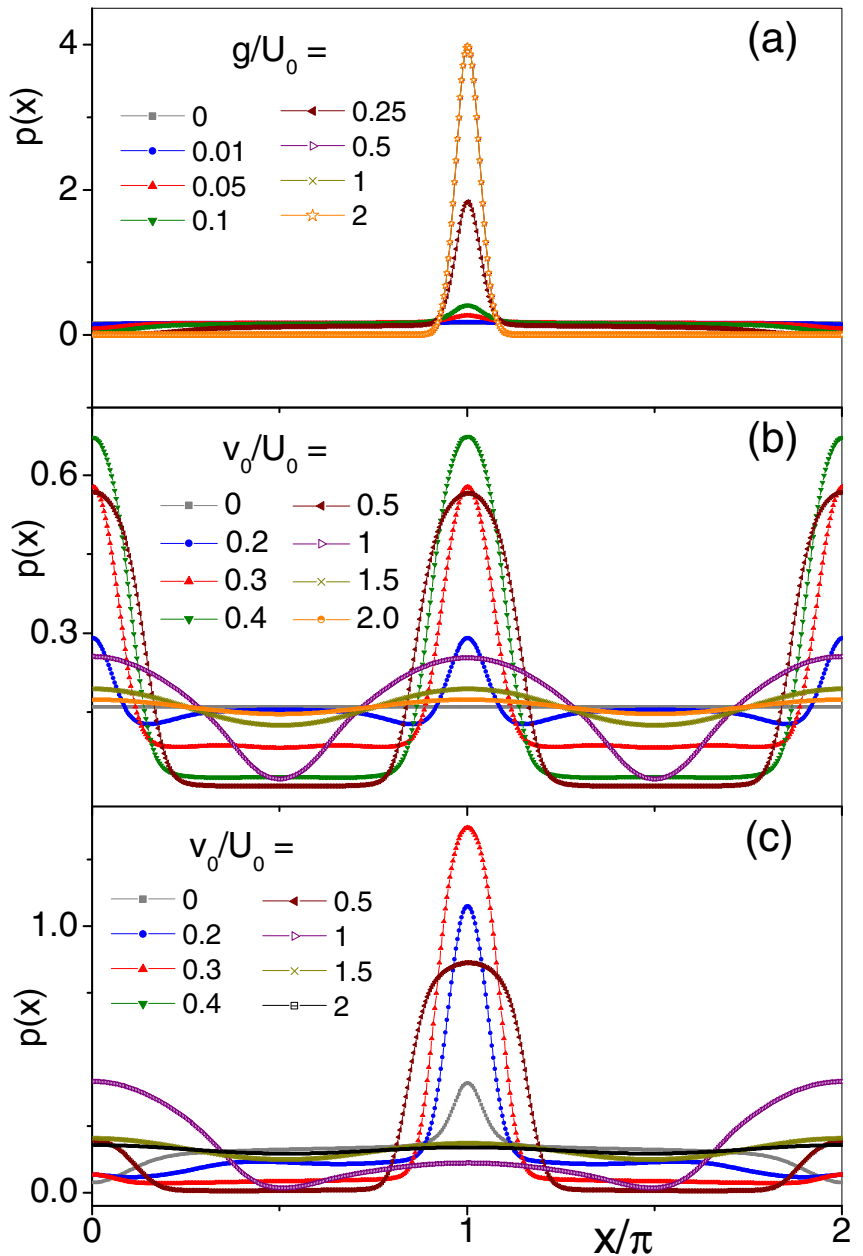

FIG. 2. Stationary longitudinal pdf's, $p(x)$, of a JP in the flow of Eq. (1): (a) $v_{0}=0$ and different $g$; (b) $g=0$ and (c) $g=0.1$ for different $v_{0}$ (see legends). Other simulation parameters are $D_{0}=$ $D_{\theta}=0.01 ; U_{0}=1$ and $L=2 \pi$.
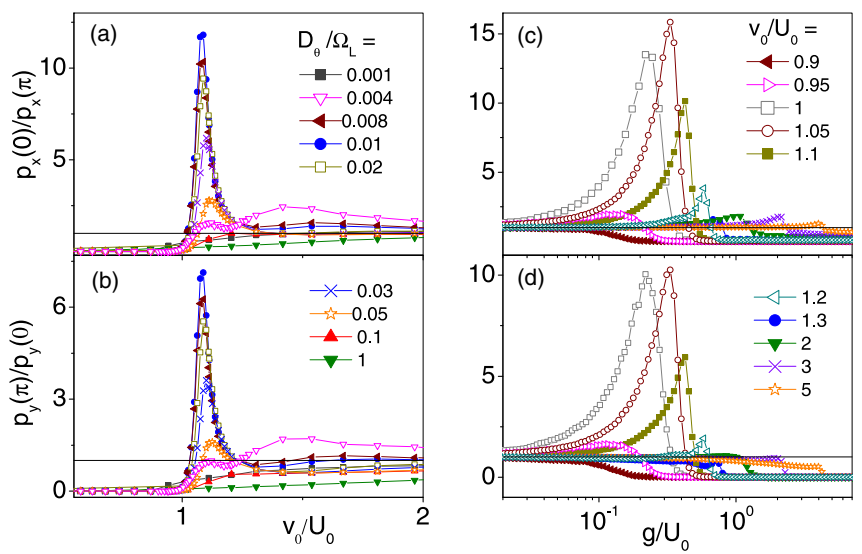

FIG. 3. Ratios of the longitudinal, $p(x)$, and transverse distributions, $p(y)$, at the boundaries of the convection rolls of Eq. (1) for (a) and (b): $g=0.4$ and different $D_{\theta}$; (c) and (d): $D_{\theta}=0.01$ and different $v_{0}$, see legends. Here $p_{x}(a)=p(x=a)$ and $p_{y}(a)=p(y=a)$ for $a=0$ and $\pi$. Other simulation parameters are $D_{0}=0.01 ; U_{0}=1$ and $L=2 \pi$, with $\Omega_{L}=1$.
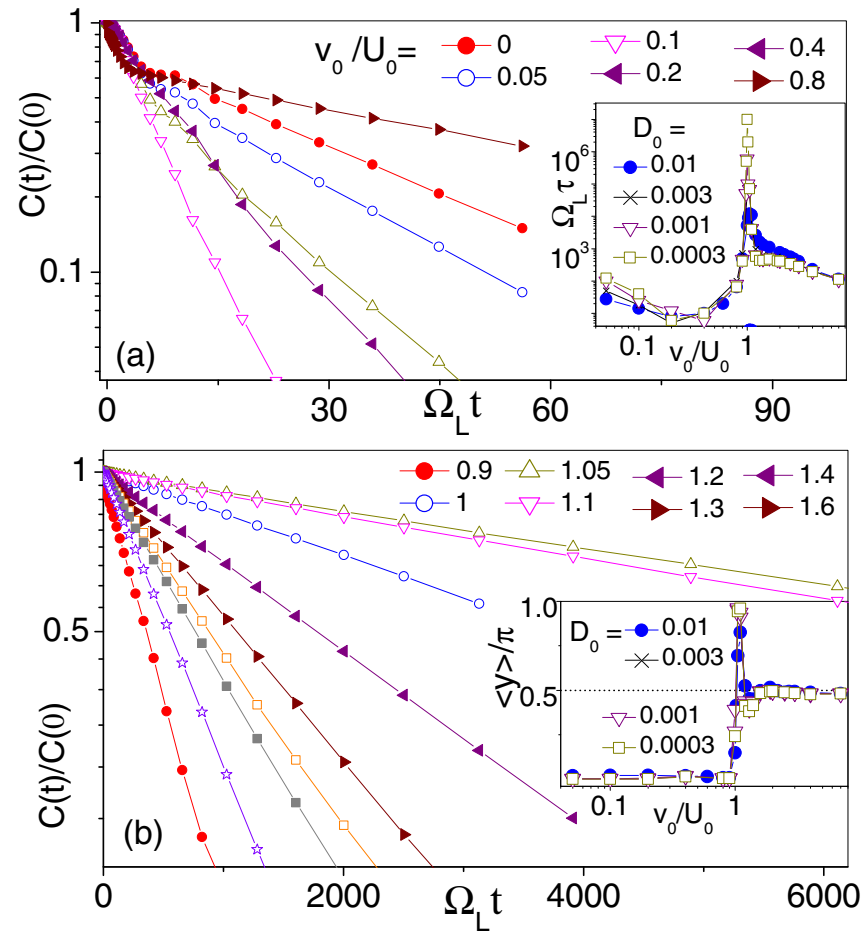

FIG. 4. Subtracted autocorrelation function $C(t)=\langle y(t) y(0)\rangle-$ $\langle y\rangle^{2}$ for different $v_{0}$. The fitted exponential decay times, $\tau$ (in units of $\Omega_{L}^{-1}$ ), and $\langle y\rangle$ (in units of $L / 2$ ) vs. $v_{0} / U_{0}$ are plotted, respectively, in the top and bottom insets for different $D_{0}$. The values of $C(0)$ are reported in the SM [29]. If not given in the legends, then the simulation parameters are $g=0.4, D_{0}=D_{\theta}=0.01 ; U_{0}=1$ and $L=2 \pi$, with $D_{L}=\Omega_{L}=1$.

at the bottom of the ascending convective flows, $p_{x}(\pi)$, and at the lower edge of the array, $p_{y}(0)$. These are the regions where, under the combined action of gravity and advection [21], a passive JP would sojourn most of the time [Fig. 2(a)]. This picture, which also applies to active JPs with low selfpropulsion speeds, gets suddenly reversed for $v_{0} \gtrsim U_{0}$. As seen in Fig. 1, the particle populates the upper edge, $p_{y}(\pi)>$ $p_{y}(0)$, in correspondence with the descending convective flows, $p_{x}(0)>p_{x}(\pi)$, as if its apparent weight had changed sign. This instance of negative response to an external bias is clearly an effect of advection. It disappears when advection grows negligible with respect to self-propulsion, $v_{0} \gg U_{0}$ in Figs. 1(a) and 1(b), and gravity, $g \gg U_{0}$ in Figs. 1(c) and 1(d). Accordingly, the ratios of Fig. 3 tend respectively to 1 and 0 .

We already noticed that such a dynamical reversal seems to be characterized by a surprisingly long time persistence [Fig. 1(f)]. To investigate this issue, in Fig. 4 we plotted the normalized stationary autocorrelation functions $C(t) / C(0)$, with $C(t)=\langle y(t) y(0)\rangle-\langle y\rangle^{2}$, for increasing values of the self-propulsion speed. The $v_{0}$ dependence of $\langle y\rangle=\langle y(0)\rangle$, inset of Fig. 4(b), is a quantifier of the "negative gravity" effect detected for $v_{0} \sim U_{0}$. All $C(t)$ curves decay exponentially, $\lim _{t \rightarrow \infty} C(t) / C(0) \propto e^{-t / \tau}$, with fitting time constant, $\tau$, reported in the inset of Fig. 4(a). For $v_{0}<U_{0}$, Fig. 4(a), the $C(t)$ curves keep memory of the convective circulation, in the form of damped oscillations for $\Omega_{L} t \lesssim 1$ [30]; for $v_{0}>U_{0}$, Fig. 4(b), the exponential tails dominate. Remarkable is the 
dependence of $\tau$ on $v_{0}: \tau$ is of the order of the rotational diffusion time, $\tau_{\theta}$, for low and very high self-propulsion speeds but goes through a huge maximum, orders of magnitude larger than $\tau_{\theta}$, in coincidence with the "negative gravity" peaks of Fig. 3. In conclusion, under suitable self-propulsion conditions, heavy active JPs float at the top of the convection array.

Discussion. As reported so far, key EB ingredients are self-propulsion, gravity, and advection. How this mechanism takes place is illustrated by the trajectory samples in Figs. 1(e) and 1(f) and the Supplemental Material (SM) [29]. For $g>g_{c}$, an active JP diffuses most of the time pressed against the lower array edge, $y=0$, and subjected to the (slowly) fluctuating horizontal drag, $v_{0} \cos \theta$, i.e., pinned by the effective tilted washboard potential $V_{e}(x)=V_{0}(x)-x v_{0} \cos \theta$. On increasing $v_{0}$, with $v_{0}<U_{0}$, the minima of $V_{e}(x)$ shift right (left) from the centers of ascending flows ( $x=\pi$ in Figs. 1 and 2). When taking stochastic averages over times much larger than $\tau_{\theta}$, this causes a broadening of the $p(x)$ peaks, see Fig. 2. On a shorter timescale, due to the rotational fluctuations, $\xi_{\theta}(t)$, the JP occasionally propels itself away from the array bottom, subjected to vertical advection, $u_{y}>0$, and shear torque, $\Omega_{\alpha}(x, y)$. This is most likely to happen at the turning points of $V_{e}(x), V_{0}(x) \mp x v_{0}\left(x=\pi\right.$ for $v_{0} \rightarrow 0$ and $x= \pm \pi / 2$ for $v_{0} \rightarrow U_{0}$ in Fig. 1). However, as the $V_{e}(x)$ turning points shift sidewise, with increasing $v_{0} u_{y}$ gets weaker and $\Omega_{\alpha}(x, y)$ stronger; accordingly, the trajectories in Fig. 1(e) soon bend downwards and the particle circulation inside the rolls is suppressed.

An abrupt change occurs when we set $v_{0}$ slightly above $U_{0}$. Self-propulsion is now strong enough not only to horizontally depin the particle from the bottom edge potential, $V_{0}(x)$, but also to propel it to the top. However, quite surprisingly, the apparent weight, $g$, does not suffice to make the particle return back to the bottom. This happen because, as gravity drags the particle downward across the $\psi(x, y)$ streamlines [25], the shear torque, $\Omega_{\alpha}(x, y)$, turns the self-propulsion velocity, $\mathbf{v}_{0}$, upwards again. This results in a sort of "sling mechanism" that traps the particle in the upper regions of the convection rolls [Figs. 1(c) and 1(f)].

Contrary to Refs. [26,27], the sling mechanism introduced here is not due to inertia, but rather to the shear torque acting on the active particle. Moreover, it explains the exceedingly long persistence time of the JP at the top of the array, where it appears to be steadily floating against gravity. Indeed, the fitting time, $\tau$ [inset of Fig. 4(a)], quantifies the effectiveness of such a dynamical trap. Under optimal conditions, it can grow orders of magnitude larger than the largest timescale in the model Eqs. (2) [i.e., $\tau_{\theta}$ in the Pe $\gg 1$ regime considered in the present report], very sensitive to the noise strength $D_{0}$ [29]. In the inset of Fig. 4(a) we notice immediately that, on decreasing $D_{0}$, the $\tau$ peaks shoot up, while shifting toward $v_{0}=U_{0}$.

We now briefly discuss the robustness of EB versus all model parameters (see the SM [29] for more details):

(i) Self-propulsion speed, $v_{0}$. As anticipated above, for $v_{0}>U_{0}>g$, self-propulsion dominates over both gravity and advection, so that the upside-down symmetry of the array is restored. Therefore, EB is restricted to values of $v_{0}$ larger than but relatively close to $U_{0}$ [Figs. 3(a) and 3(b)].

(ii) Shear torque coefficient, $\alpha$. So far we adopted Faxén's prescription, $\alpha=1[24,31]$, which means that inside the convection rolls the drag vectors, $\mathbf{v}_{0}$ and $\mathbf{u}$, tend to be antiparallel. This was tacitly assumed in our discussion of trajectory localization at the lower and upper edges, respectively, for $v_{0} \lesssim U_{0}$ and $v_{0} \gtrsim U_{0}$, see Figs. 1(e) and 1(f). Lowering $\alpha$, or worse changing its sign, clearly suppresses the dynamical trapping mechanism responsible for EB.

(iii) Apparent weight, $g$. EB is detectable for all $g$ values up to a certain maximum; it vanishes only when $g$ prevails over the combined action of self-propulsion and advection, that is for $g \sim v_{0}$ at large $v_{0}$ [Figs. 3(c) and 3(d)].

(iv) Rotational noise strength, $D_{\theta}$. The noise $\xi_{\theta}(t)$ makes $\mathbf{v}_{0}$ change direction with time constant $\tau_{\theta}=1 / D_{\theta}$. Figures 3(a) and 3(b) clearly show that the onset of EB is not affected by $D_{\theta}$, while the magnitude of the effect is. EB is suppressed both for too-large $D_{\theta}$, when the persistence length, $l_{\theta}$, is much shorter than the roll size, $L$, and too small $D_{\theta}$, when the timescale of thermal noise activated diffusion along the array bottom [modeled by $V_{e}(x)$ ] is shorter than $\tau_{\theta}$. EB is clearly detectable for a relatively wide range of intermediate $D_{\theta}$ values, with $D_{\theta}<\Omega_{L}$.

Conclusions. We have shown that a heavy JP constrained to a linear array of convection rolls can rise to the top due to the combined action of gravity and advection, an effect we termed "enhanced buoyancy." The advective torque is responsible for the underlying "sling mechanism," which maintains the particle floating on the surface for exceedingly long time intervals. The "sling mechanism" reported here is rather selective with respect to the JP's mass density and self-propulsion properties, which suggests potential applications at both microfluidic and environmental scales.

Acknowledgments. Y.L. is supported by the NSF China under Grants No. 11875201 and No. 11935010. P.K.G. is supported by SERB Start-up Research Grant (Young Scientist) No. YSS/2014/000853 and the UGC-BSR Start-Up Grant No. F.30-92/2015.
[1] R. Kubo, Brownian motion and nonequilibrium statistical mechanics, Science 233, 330 (1986).

[2] I. I. Blekhman, Vibrational Mechanics (World Scientific, Singapore, 2000).

[3] B. Apffel, F. Novkoski, A. Eddi, and E. Fort, Floating under a levitating liquid, Nature (London) 585, 48 (2020).

[4] G. H. Wolf, Dynamic Stabilization of the Interchange Instability of a Liquid-Gas Interface, Phys. Rev. Lett. 24, 444 (1970).
[5] A. Rosato, K. J. Strandburg, F. Prinz, and R. H. Swendsen, Why the Brazil Nuts are On Top: Size Segregation of Particulate Matter by Shaking, Phys. Rev. Lett. 58, 1038 (1987).

[6] P. Gajjar, C. G. Johnson, J. Carr, K. Chrispeels, J. M. N. T. Gray, and P. J. Withers, Size segregation of irregular granular materials captured by time-resolved 3D imaging, Sci. Rep. 11, 8352 (2021). 
[7] O. Bénichou, P. Illien, G. Oshanin, A. Sarracino, and R. Voituriez, Microscopic Theory for Negative Differential Mobility in Crowded Environments, Phys. Rev. Lett. 113, 268002 (2014).

[8] R. K. P. Zia, E. L. Praestgaard, and O. G. Mouritsen, Getting more from pushing less: Negative specific heat and conductivity in nonequilibrium steady states, Am. J. Phys. 70, 384 (2002).

[9] R. Eichhorn, P. Reimann, and P. Hänggi, Brownian Motion Exhibiting Absolute Negative Mobility, Phys. Rev. Lett. 88, 190601 (2002).

[10] L. Machura, M. Kostur, P. Talkner, J. Luczka, and P. Hänggi, Absolute Negative Mobility Induced by Thermal Equilibrium Fluctuations, Phys. Rev. Lett. 98, 040601 (2007).

[11] A. Sarracino, F. Cecconi, A. Puglisi, and A. Vulpiani, Nonlinear Response of Inertial Tracers in Steady Laminar Flows: Differential and Absolute Negative Mobility, Phys. Rev. Lett. 117, 174501 (2016).

[12] W. Young, A. Pumir, and Y. Pomeau, Anomalous diffusion of tracer in convection rolls, Phys. Fluids A 1, 462 (1989).

[13] S. Jiang and S. Granick (Eds.), Janus Particle Synthesis, Self-assembly and Applications (RSC Publishing, Cambridge, 2012).

[14] A. Walther and A. H. E. Müller, Janus particles: Synthesis, selfassembly, physical properties, and applications, Chem. Rev. 113, 5194 (2013).

[15] M. C. Marchetti, J. F. Joanny, S. Ramaswamy, T. B. Liverpool, J. Prost, M. Rao, and R. A. Simha, Hydrodynamics of soft active matter, Rev. Mod. Phys. 85, 1143 (2013).

[16] J. Elgeti, R. G. Winkler, and G. Gompper, Physics of microswimmers, single particle motion and collective behavior: A review, Rep. Prog. Phys. 78, 056601 (2015).

[17] W. M. Durham, E. Climent, and R. Stocker, Gyrotaxis in a Steady Vortical Flow, Phys. Rev. Lett. 106, 238102 (2011).

[18] G. I. Taylor, On the decay of vortices in a viscous fluid, Philos. Mag. 46, 671 (1923).
[19] S. Chandrasekhar, Hydrodynamic and Hydromagnetic Stability (Oxford University Press, New York, 1967).

[20] E. Bodenschatz, W. Pesch, and G. Ahlers, Recent developments in Rayleigh-Bénard convection, Annu. Rev. Fluid Mech. 32, 709 (2000).

[21] Y. Li, Q. Yin, F. Marchesoni, T. Debnath, and P. K. Ghosh, Advection-enhanced diffusion in biased convection arrays, Phys. Rev. E 103, L030106 (2021).

[22] Y. Li, L. Li, F. Marchesoni, D. Debnath, and P. K. Ghosh, Active diffusion in convection rolls, Phys. Rev. Research 2, 013250 (2020).

[23] P. K. Ghosh, F. Marchesoni, Y. Li, and F. Nori, Active particle diffusion in convection roll arrays, Phys. Chem. Chem. Phys. 23, 11944 (2021).

[24] A. Zöttl and H. Stark, Emergent behavior in active colloids, J. Phys.: Condens. Matter 28, 253001 (2016).

[25] M. I. Yudine, Physical Considerations on Heavy Particle Diffusion, in Advances in Geophysics, Vol. 6 (Academic Press, New York, 1959), pp. 185.

[26] G. Falkovich, A. Fouxon, and M. G. Stepanov, Acceleration of rain initiation by cloud turbulence, Nature (London) 419, 151 (2002).

[27] G. P. Bewley, E. W. Saw, and E. Bodenschatz, Observation of the sling effect, New J. Phys. 15, 083051 (2013).

[28] P. E. Kloeden and E. Platen, Numerical Solution of Stochastic Differential Equations (Springer, Berlin, 1992).

[29] See Supplemental Material at http://link.aps.org/supplemental/ 10.1103/PhysRevResearch.3.L032065 for additional simulation data.

[30] Q. Yin, Y. Li, F. Marchesoni, T. Debnath, and P. K. Ghosh, Exit times of a Brownian particle out of a convection roll, Phys. Fluids 32, 092010 (2020).

[31] C. Torney and Z. Neufeld, Transport and Aggregation of SelfPropelled Particles in Fluid Flows, Phys. Rev. Lett. 99, 078101 (2007). 